\section{Cadmium bioaccumulation in European flat oysters (Ostrea edulis) from Middle Adriatic Sea (San Benedetto del Tronto district, Italy)}

\author{
Cesare Ciccarelli, Angela Marisa \\ Semeraro, Alessandra Aliventi, Vittoria \\ Di Trani, Piero Capocasa
}

Servizio Veterinario di Igiene degli Alimenti di Origine Animale - ASUR

Marche, Area Vasta n. 5, San Benedetto del Tronto, Italy

\section{Abstract}

Bivalve molluscs represent an important source of cadmium exposure in humans, in particular oysters, because of their high filter feeding capability and high concentration of metal-binding metallothionein in tissues. In this study the authors investigated the difference in cadmium bioaccumulation in European flat oysters harvested from production areas in the district of San Benedetto del Tronto (Ascoli Piceno province, Italy), as a function of their origin (farming or natural beds) and the time of gathering. The beds lie 3 $\mathrm{nm}$ off-shore at a depth of $20-40 \mathrm{~m}$ and are collected by dredging. In the farms, baskets are suspended in the water column $2.5-3 \mathrm{~nm}$ offshore at a depth of $4 \mathrm{~m}$. The authors analysed the results of cadmium monitoring plan carried out in oyster natural beds for a total of 15 samples collected from 2004 to 2012 and in two oyster farms for a total of 11 samples from 2009 to 2012. Although the few data did not allow to find a significant statistical association, they suggested two findings: i) cadmium concentration in oysters from natural beds seemed to be lower than in farmed oysters; and ii) in farmed oysters cadmium concentration even exceeded allowed maximum level for human consumption, in particular in autumn. The vertical stratification in the water column of phytoplankton and a cadmium dilution at oyster gonadal maturation might cause changes in oyster cadmium accumulation.

\section{Introduzione}

La principale fonte di esposizione al cadmio, per i non fumatori, è rappresentata dagli alimenti e, tra questi, un contributo non trascurabile è attribuibile ai molluschi bivalvi, ed in particolare alle ostriche. Tale caratteristica dipende dall'elevata capacità filtrante di questi bivalvi e dalla particolare concentrazione nei loro tessuti di metallotioneine, proteine ad elevata affinità per gli ioni metallici. Le norme per la sicurezza alimentare ne fissano tenori massimi consentiti nei molluschi bivalvi e prevedono piani di monitoraggio nelle zone di produzione. Il Servizio Veterinario di Igiene degli Alimenti di Origine Animale di San Benedetto del Tronto effettua dal 2004 il monitoraggio dei livelli di cadmio nelle ostriche dei banchi naturali, posti oltre le tre miglia dalla costa, raccolte mediante lo strascico, e dal 2009 il monitoraggio nelle ostriche provenienti da due allevamenti che praticano l'ingrasso, su filari longline, dei soggetti raccolti da banchi naturali. Diversi autori hanno evidenziato differenze nella concentrazione del contaminante, per ostriche provenienti dallo stesso ambiente, in funzione di diversi fattori: età delle ostriche (Rasmussen et al., 2007), stagionalità (PaezOsuna et al., 1995), disposizione lungo la colonna d'acqua (Strady et al., 2011). Con il presente lavoro si intende fornire una valutazione preliminare dei livelli di cadmio accumulati nelle ostriche provenienti dalle aree di raccolta del distretto di San Benedetto del Tronto, attraverso l'analisi dei risultati del monitoraggio, per individuare eventuali differenze in funzione dell'origine delle ostriche e del periodo di raccolta.

\section{Materiali e Metodi}

Sono stati presi in considerazione i seguenti gruppi di ostriche: i) da banchi naturali, posti ad una profondità tra i 20 e i 40 m oltre le $3 \mathrm{~nm}$ dalla costa, e raccolte mediante strascico, per un totale di 15 campioni prelevati tra il 2004 ed il 2012; ii) da 2 allevamenti, localizzati a circa $2,5-3 \mathrm{~nm}$ dalla costa, che praticano l'ingrasso di soggetti raccolti da banchi naturali mediante lanterne sospese su filari long-line,
Correspondence: Cesare Ciccarelli, Servizio Veterinario di Igiene degli Alimenti di Origine Animale - ASUR Marche, Area Vasta n. 5, piazza Nardone 19, 63074 San Benedetto del Tronto (AP), Italy.

Tel. +39.0735 .7937474 - Fax: +39.0735 .793529 .

E-mail: cesare.ciccarelli@sanita.marche.it

Key words: Flat oyster, Ostrea edulis, cadmium bioaccumulation, Seafood.

Received for publication: 10 April 2013. Revision received: 24 October 2013 Accepted for publication: 29 October 2013.

This work is licensed under a Creative Commons Attribution 3.0 License (by-nc 3.0).

CC Copyright C. Ciccarelli et al., 2014

Licensee PAGEPress, Italy

Italian Journal of Food Safety 2014; 3:1672

doi:10.4081/ijfs.2014.1672

ad una profondità di circa 4 metri, per un totale di 11 campioni prelevati tra il 2009 ed il 2012.

I molluschi, prelevati in singola aliquota (4 kg circa) e conferiti vivi e vitali al laboratorio, sono stati sottoposti ad analisi secondo procedura interna, mediante ICP-MS, presso la sezione di Ancona dell'I.Z.S. Umbria e Marche. Nella Tabella 1 sono riportati i valori ottenuti per i due gruppi di ostriche; nelle Tabelle 2 e 3 , rispettivamente per i banchi naturali e per gli allevamenti, gli stessi dati sono stati raggruppati su base mensile. Le relative distribuzioni sono rappresentate graficamente nelle Figure 1, 2 e 3 mediante il metodo Box and Whiskers secondo Tuckey, utilizzando per il valore adiacente superiore la differenza interquartile ( $3^{\circ}$ quartile- $1^{\circ}$ quartile) moltiplicata per 1,5 .

\section{Risultati e Discussione}

I risultati disponibili, pur ancora basati su dati insufficienti per una valutazione significativa dal punto di vista statistico, permettono di delineare due precise tendenze. Da una parte la concentrazione di cadmio nelle ostriche da banchi naturali appare inferiore a quel-

Tabella 1. Valori di cadmio nei due gruppi ostriche (espressi in $\mathrm{mg} / \mathrm{kg}$ ).

\begin{tabular}{|c|c|c|c|c|c|c|c|c|c|c|c|c|c|c|c|}
\hline Gruppi di ostr & & & & & & & & i di ca & Amio & & & & & & \\
\hline Banchi naturali & 0.620 & 0.950 & 0.810 & 0.700 & 0.714 & 0.694 & 0.570 & 0.524 & 0.604 & 0.492 & 0.564 & 0.646 & 0.741 & 0.649 & 0.613 \\
\hline Allevamenti & 0.687 & 0.905 & 1.331 & 0.908 & 0.589 & 0.685 & 0.962 & 0.776 & 1.733 & 0.722 & 1.000 & - & - & - & - \\
\hline
\end{tabular}


la delle ostriche allevate (Figura 1), nelle quali, in alcuni casi, è stato addirittura superato il limite consentito per il consumo umano (1 $\mathrm{mg} / \mathrm{kg}$ peso fresco). Questo risultato, in linea con quanto riscontrato da altri autori, potrebbe essere attribuito alla diversa localizzazione delle ostriche nella colonna d'acqua: quelle allevate, sospese a 4 metri di profondità, si alimenterebbero prevalentemente di fitoplancton, più contaminato dal cadmio, mentre le ostriche da banchi naturali, disposte sul fondo, utilizzerebbero prevalentemente microalghe bentoniche (Strady et al., 2011; Rodney et al., 2007); inoltre la più lenta attività metabolica per le ostriche disposte sul fondo suggerirebbe una più ridotta velocità di filtrazione e, quindi, una minore velocità nell'accumulo del cadmio (Le Moullac et al., 2007).
Dall'altra parte, appare evidente la tendenza ad un andamento stagionale della concentrazione di cadmio sia per i banchi naturali (Figura 2) che per le ostriche allevate (Figura 3) dove le differenze risultano più marcate $\mathrm{e}$, nel periodo autunnale, si riscontrano valori massimi, anche oltre il limite consentito per il consumo umano.

L'andamento stagionale, in accordo con Paez-0suna et al. (1995), potrebbe essere messo in relazione non tanto con repentini fenomeni di accumulo e successiva detossificazione, ma con il grado di sviluppo delle gonadi nei diversi periodi dell'anno. Queste incidono sensibilmente sul peso totale del corpo del mollusco e causerebbero una diluizione della concentrazione di cadmio nel periodo di massimo sviluppo oppure una conseguente con- centrazione successivamente all'emissione dei gameti.

\section{Conclusioni}

Questi primi risultati, che necessitano di essere confermati da ulteriori dati, mostrano come il livello di concentrazione del cadmio nei tessuti delle ostriche (Ostrea edulis) possa subire fluttuazioni non direttamente legate a particolari fonti di contaminazioni ma allo stato fisiologico dei molluschi e alle modalità di apporto nutritivo, provocando comunque rilevanti ripercussioni in tema di sicurezza alimentare.

Tabella 2. Valori di cadmio nei banchi naturali, raggruppati su base mensile (espressi in $\mathrm{mg} / \mathrm{kg}$ ).

\begin{tabular}{|c|c|c|c|c|c|c|c|c|}
\hline Gruppi di ostriche su base mensile & & & & i di ce & & & & \\
\hline Gennaio & 0.620 & - & - & - & - & - & - & - \\
\hline Marzo & 0.950 & - & - & - & - & 0.646 & - & 0.613 \\
\hline Aprile & 0.810 & 0.700 & 0.694 & - & 0.492 & - & - & - \\
\hline Luglio & - & - & 0.570 & - & - & - & 0.649 & - \\
\hline Ottobre & 0.714 & - & 0.524 & - & 0.564 & - & - & - \\
\hline Novembre & - & - & - & 0.604 & - & 0.741 & - & - \\
\hline
\end{tabular}

Tabella 3. Valori di cadmio negli allevamenti raggruppati, su base mensile (espressi in $\mathrm{mg} / \mathrm{kg}$ ).

\begin{tabular}{lcccccc} 
Gruppi di ostriche su & \multicolumn{7}{c}{ Valori di cadmio } \\
base mensile & - & - & - & - & - & 0.722 \\
Gennaio & - & - & 0.589 & 0.685 & 0.776 & - \\
Marzo & 0.687 & - & - & - & - & - \\
\hline Aprile & - & - & - & - & - & 1.000 \\
Luglio & 0.905 & - & 0.962 & - & 1.733 & - \\
Settembre & 1.331 & - & - & - & - & - \\
Ottobre & 0.908 & - & - & - & - & - \\
\hline Novembre & & & & &
\end{tabular}

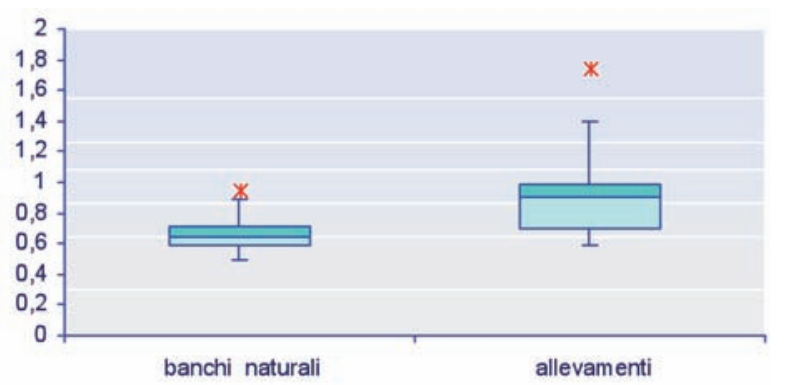

* Min Outlier * Max Outlier

Figura 1. Distribuzione dei risultati per le ostriche da allevamento e da banchi naturali.

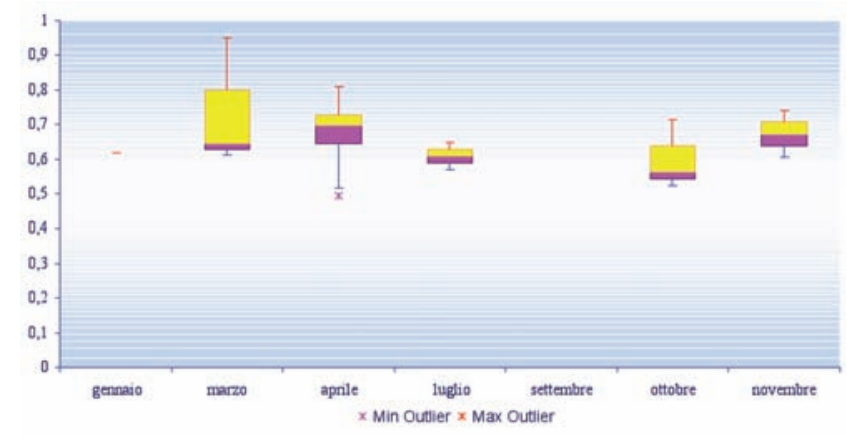

Figura 2. Ostriche da banchi naturali: distribuzione su base mensile.

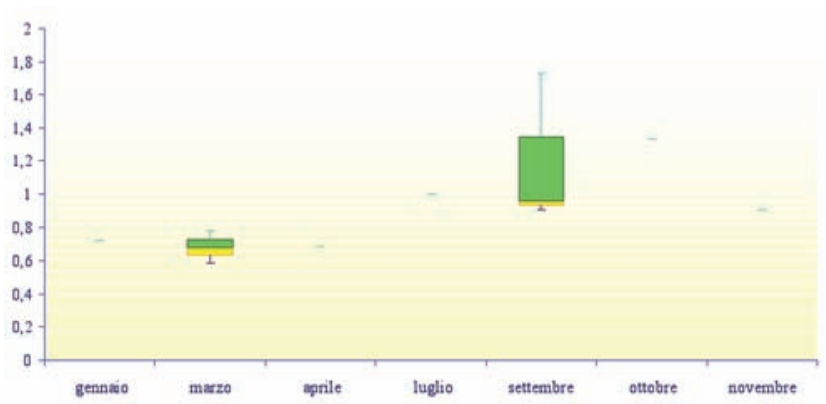

Figura 3. Ostriche allevate: distribuzione su base mensile. 
Lopea JI, 1995. Trace metal concetrations in relation to season and gonadal maturation in the oyster Crassostrea iridescens. Mar Environ Res 40:19-31.

Le Moullac G, Fleury PG, Le Coz JR, Moal J, Samain J-F, 2007. Effect of sediment nearness on the metabolic enzyme activity and energy state of the oyster Crassostrea gigas. Disponibile al sito: http:/archimer. ifremer.fr/doc/2007/publication-2803.pdf

Paez-Osuna F, Frias-Espericuela MG, Osuna-
Rasmussen RS, Morissey MT, Cheney D, 2007. Effect of age and tissue weight on the cadmium concentration in pacific oysters (Crassostrea gigas). J Shellfish Res 26:173-9.

Rodney E, Herrera P, Luxama J, Boykin M, Crawford A, Carrol MA, Catapane EJ, 2007.
Bioaccumulation and tissue distribution of Arsenic, Cadmium, Copper and Zinc in Crassostrea virginica grown at two different depths in Jamaica Bay, New York. In vivo 29:16-27.

Strady E, Blanc G, Baudrimont M, Schäfer J, Robert S, Lafon V, 2011. Roles of regional hydrodynamic and trophic contamination in cadmium bioaccumulation by Pacific oysters in the Marennes-Oléron Bay (France). Chemosphere 84:80-90. 